\title{
SUBSTRATOS NA PRODUÇÃO DE MUDAS DE ESPÉCIES ARBÓREAS NATIVAS PARA ARBORIZAÇÃO URBANA
}

\author{
Fábio Gomes de Oliveira1; Mário Augusto Gonçalves Jardim² ${ }^{3}$
}

\section{RESUMO}

Os impactos antrópicos na Amazônia têm ocasionado a degradação ambiental em diversos ecossistemas, sendo o cultivo e o plantio de espécies arbóreas nativas uma estratégia viável para a restauração ambiental em curto prazo. O objetivo deste estudo foi avaliar o efeito de substratos no tempo de emergência e na quantidade de sementes germinadas de Ceiba Pentandra (L.) Gaertn. (Malvaceae), Ficus maxima Mill. (Moraceae), Matisia paraensis Huber (Malvaceae) e Inga bourgonii (Aubl.) DC. (Fabaceae), espécies arbóreas com valor econômico, ambiental, cultural e com potencial paisagístico na região amazônica. $O$ delineamento foi em blocos casualizados com quatro tratamentos: T1- terra preta, T2- areia, T3- vermiculita e T4- terra preta $(50 \%)$ + vermiculita $(50 \%)$ e três repetições para C. Pentandra e $F$. maxima. As espécies $M$. paraensis e $I$. bourgonii foram submetidas aos tratamentos T1, T2, T3 e T4- terra preta (50\%) + areia (50\%), sendo avaliado durante 60 dias. Foi aplicado o Teste de Tukey ao nível de $5 \%$ de probabilidade com o auxílio do programa Bioestat 5.0. Os substratos não influenciaram no tempo de emergência, enquanto que a maior quantidade de sementes germinadas foi proporcionada pela vermiculita que atua como excelente retentora de água e com ótima qualidade estrutural.

Palavras-Chaves: Plantio de Mudas; Restauração Ambiental;Conservação de espécies nativas.

\section{SUBSTRATES IN SEEDLING PRODUCTION OF NATIVE TREE SPECIES FOR URBAN LANDSCAPE}

\section{ABSTRACT}

The human impacts in the Amazon have caused environmental degradation in many ecosystems, and the cultivation and planting of native tree species a viable strategy for reforestation in the short term, contributing to environmental restoration. The objective of this research was to evaluate the effect of substrate on the emergency time and the amount of germinated seeds of Ceiba pentandra (L.) Gaertn. (Malvaceae), Ficus maxima Mill. (Moraceae), Matisia paraensis Huber (Malvaceae) and Inga bourgonii (Aubl.) DC. (Fabaceae), tree species with economic value, environmental, cultural and scenic potential in the Amazon region. The experimental design was randomized blocks with four treatments (T1-black earth, T2-sand, T3-vermiculite and T4-black earth (50\%) + vermiculite (50\%). The experiment was evaluated to 60 days. For the data obtained was applied Tukey test at $5 \%$ probability with the aid of BioStat 5.0. The substrates had no influence in time of emergency, while the largest number of germinated seeds was provided by vermiculite and acts as retaining excellent water quality and has great structure.

Keywords: Ecosystems, Planting Seedlings, Environmental Restoration.

\footnotetext{
${ }^{1}$ Bolsista PIBIC/CNPq. Museu Paraense Emilio Goeldi. Coordenação de Botânica, Belém/Pará: fg_oliveiraufra@yahoo.com.br

2 Pesquisador Titular. Museu Paraense Emilio Goeldi.Coordenação de Botânica. Belém/Pará: jardim@museu-goeldi.br

${ }^{3}$ recebido em 08.07.2013 e aceito para publicação em 15.09.2013
} 


\section{INTRODUÇÃO}

A germinação corresponde a uma etapa na vida da semente que é influenciada por fatores ambientais e genéticos durante o seu desenvolvimento (LOPES; PEREIRA, 2005). No caso de alterações neste processo, a emergência da planta sofrerá consequências, pois, é um fenômeno primordial para o seu estabelecimento, e caso apresente atraso, poderá ocasionar o menor crescimento da parte aérea e do sistema radicular (Merotto Junior et al., 1999).

O substrato influencia no comportamento germinativo das espécies de maneira não uniforme, de forma que algumas são mais exigentes e com melhor desempenho em apenas um tipo de substrato ou pela mistura deste. A prática de cultivar plantas utilizando substratos objetiva a determinação do melhor padrão vital de cultivo no menor tempo, nesse sentido, a sua escolha ocorre em função da facilidade e da eficiência do uso e do tipo de semente, pois, é onde o sistema radicular contribuirá no crescimento da parte aérea até o transplantio (Braga Junior et al. 2010; Jabur; Martins, 2002). Para Souza et al. (2001) é o responsável pelo fornecimento de nutrientes, disponibilidade e capacidade de retenção de água.

O uso intenso de espécies nativas no setor madeireiro e a degradação de ecossistemas vêm ocasionando o desaparecimento de muitas espécies com potencial paisagístico, madeireiro ou de preservação (Varela et al. 2005). Diante de tal realidade, são necessários estudos sobre a propagação vegetativa de espécies arbóreas com ênfase no tempo de emergência, como alternativa para minimizar em curto prazo os impactos ambientais ocasionados pela ação antrópica.

Dentre as inúmeras espécies com potencial econômico na região amazônica foram escolhidas: Ceiba pentandra (L.) Gaertn., considerada uma das maiores árvores da America tropical que atualmente está ameaçada de extinção em decorrência da extração predatória (Sousa et al., 2000); Ficus maxima Mill., frutífera arbórea cujo representa importância alimentar para a fauna e para arborização de parques e vias urbanas (Carauta \& Diaz, 2002); Matisia paraensis Huber árvore de médio porte com copa ovalada e densa, esta espécie fornece fibras resistentes com uso para fabricação de papel (Corrêia, 1984) e Inga bourgonii (Aubl.) DC., frutífera que atinge até $13 \mathrm{~m}$ de altura e pode ser utilizada para fins paisagísticos (Sousa et al., 2011).

Para a utilização de espécies arbóreas amazônicas no paisagismo, a produção de mudas é uma etapa fundamental e, o 
plantio e a reposição destas, contribuem na preservação dos remanescentes existentes.

O objetivo deste estudo foi avaliar a influência de diferentes substratos no tempo de emergência e na quantidade de sementes germinadas de Ceiba Pentandra; Ficus máxima; Matisia paraensis e Inga bourgonii.

\section{MATERIAL E MÉTODOS}

\section{Área de coleta}

A coleta de frutos foi realizada nos meses de agosto de 2012 a março de 2013 em cinco matrizes por espécie, localizadas na Área de Proteção Ambiental Ilha do Combú, Belém, Pará, conforme a autorização concedida para coleta de material botânico pela Secretaria de Estado e Meio Ambiente (SEMA/n ${ }^{\circ}$ 017/2010).

\section{Experimentação}

Os frutos maduros foram coletados diretamente das plantas, depositados em sacos plásticos de $30 \mathrm{~kg}$ e encaminhados ao laboratório de Ecologia vegetal da Coordenação de Botânica do Museu Paraense Emílio Goeldi, onde as sementes foram extraídas manualmente. As sementes foram lavadas em água corrente e secadas ao sol em bandejas plásticas durante 4 dias. Posteriormente, instalou-se um experimento em blocos casualizados com quatro tratamentos sendo: T1- terra preta; T2- areia; T3vermiculita e T4- terra preta (50\%) + vermiculita (50\%) e três repetições para $C$. Pentandra (1092 sementes) e F. maxima (396 sementes) e M. paraensis (180 sementes) e $I$. bourgonii (204 sementes) submetidas aos tratamentos T1, T2, T3 e T4- terra preta (50\%) + areia (50\%).

O experimento foi conduzido em bancada suspensa em temperatura ambiente (condições de sombreamento) na Coordenação de Botânica do Museu Paraense Emilio Goeldi no período de setembro/2012 a janeiro/2013 e avaliado durante 60 dias com observações diárias. Foram consideradas emergidas as plantas que apresentavam cotilédones acima do substrato.

Foi calculada a média, variância, desvio padrão e o erro padrão e aplicado o Teste de Tuckey ao nível de $5 \%$ de probabilidade com auxílio do Programa BioEstat 5.0. (Ayres et al., 2007) e a taxa de emergência foi calculada por percentagem. 


\section{RESULTADOS}

No processo de emergência para Ceiba pentandra foram constatadas 181 plantas no T3 (66,3\%), 144 no T2 (52,75\%), 54 no T4 (19,78\%) e 18 plantas no T1 (6,59\%). No total emergiram 397 plantas, o que representou 36,35\%. O pico de emergência ocorreu inicialmente na mistura com terra preta e vermiculita no $48^{\circ}$ dia, na terra preta no $54^{\circ}$ dia, na vermiculita no $57^{\circ}$ dia e na areia no $58^{\circ}$ dia.

$\mathrm{Na}$ Tabela 1 verifica-se que o T3 foi responsável pela maior média no processo de emergência (60 plantas) seguido do T2 (48 plantas), portanto, estes substratos proporcionaram condições adequadas.

Tabela 1. Análise estatística do processo de emergência de plântulas de Ceiba pentandra (L.) em diferentes substratos

\begin{tabular}{ccccc}
\hline Tratamentos & T1 & T2 & T3 & T4 \\
\hline Média & 4,0 & 48 & 60 & 16,7 \\
Variância & 1,0 & 28 & 12 & 25,3 \\
Desvio padrão & 1,0 & 5,3 & 3,5 & 5,0 \\
Erro padrão & 0,58 & 3,1 & 2,0 & 2,9 \\
\hline
\end{tabular}

T1- terra preta; T2- areia; T3- vermiculita e T4- terra preta (50\%) + vermiculita (50\%).

Tabela 2. Análise estatística do processo de emergência de plântulas de Ficus maxima Mill. em diferentes substratos

\begin{tabular}{ccccc}
\hline Tratamentos & T1 & T2 & T3 & T4 \\
\hline Média & 24,7 & 5,3 & 26,7 & 22,0 \\
Variância & 6,3 & 5,3 & 6,3 & 1,0 \\
Desvio padrão & 2,5 & 2,3 & 2,5 & 1,0 \\
Erro padrão & 1,4 & 1,3 & 1,4 & 0,6 \\
\hline
\end{tabular}

T1- terra preta; T2- areia; T3- vermiculita e T4- terra preta (50\%) + vermiculita (50\%).

Para F. maxima, observou-se a emergência de 232 plantas perfazendo um total de 58,58\% sendo o T3 e T1 aqueles que promoveram a emergência de 80 e 74 plantas $(80,81 \%$ e $74,75 \%)$ respectivamente.
Conforme a Tabela 2, o T3 foi responsável pela maior média no processo de emergência (26,7 plantas) e proporcionou o maior número de plantas emergidas. 
Para M. paraensis foi observada a emergência de 43 plantas no T3 e T4 (95,56\%), seguido pelo T1 com 40 plantas $(88,89 \%)$ e 36 plantas no T2 (80\%). Os tratamentos proporcionaram a emergência de 162 plantas, representando 90\% do total.
De acordo com a Tabela 3, a maior média no processo de emergência foi obtida no T3 e no T4 correspondente a 14,3 plantas, e que portanto, promoveram o maior número de plantas emergidas.

Tabela 3. Análise estatística do processo de emergência de plântulas de Matisia paraensis Huber. em diferentes substratos

\begin{tabular}{ccccc}
\hline Tratamentos & T1 & T2 & T3 & T4 \\
\hline Média & 13,3 & 12,0 & 14,3 & 14,3 \\
Variância & 1,3 & 27,0 & 1,3 & 0,33 \\
Desvio padrão & 1,15 & 5,2 & 1,15 & 0,58 \\
Erro padrão & 0,67 & 3,0 & 0,67 & 0,33 \\
\hline
\end{tabular}

T1- terra preta; T2- areia; T3- vermiculita e T4- terra preta (50\%) + (50\%) areia.

Foi constatada a emergência de 197 plantas de $I$. bourgonii, correspondendo a $96,56 \%$ do total. O padrão inicial de emergência foi progressivo até o final do experimento, de forma que no T3 foram observadas 51 plantas (100\%), seguido pelo T4 com 50 plantas (98,03\%), T2 com
49 plantas $(96,07 \%)$ e com 47 no $T 1$ (92,15\%).

$\mathrm{Na}$ Tabela 4, verifica-se que no processo de emergência a maior média foi para o T3 (17 plantas). Contudo, o T4 (16,7 plantas) e T2 (16,3 plantas) obtiveram valores de média muito próximo ao T3, e que, portanto, favoreceram a emergência.

Tabela 4. Análise estatística do processo de emergência de plântulas de Inga bourgonii (Aubl.) DC. em diferentes substratos

\begin{tabular}{ccccc}
\hline Tratamentos & T1 & T2 & T3 & T4 \\
\hline Média & 15,7 & 16,3 & 17,0 & 16,7 \\
Variância & 2,3 & 1,3 & 0,0 & 0,3 \\
Desvio padrão & 1,5 & 1,1 & 0,0 & 0,6 \\
Erro padrão & 0,9 & 0,7 & 0,0 & 0,3 \\
\hline
\end{tabular}

T1- terra preta; T2- areia; T3- vermiculita e T4- terra preta (50\%) + (50\%) areia.

Na Tabela 5, nota-se o desempenho dos substratos no processo de emergência das espécies, onde a vermiculita foi significativamente superior comparada 
aos demais substratos, proporcionando,

espécies.

assim, o sucesso quantitativo das

Tabela 5. Média e desvio padrão do número de plantas de cinco espécies arbóreas no substratos: TP (terra preta), AR (areia), VERM (vermiculita), TP + VERM (terra preta + vermiculita) e TP + AR (terra preta + areia)

\begin{tabular}{ccccc}
\hline Espécie & TP & AR & VERM & TP + VERM \\
\hline C. pentandra & $6,00 \pm 2,00$ a & $48,00 \pm 5,29$ a & $60,33 \pm 3,21 \mathbf{b}$ & $18,00 \pm 6,00$ a \\
F. maxima & $24,67 \pm 2,52$ a & $5,33 \pm 2,31$ a & $26,67 \pm 2,52 \mathbf{b}$ & $22,33 \pm 1,15$ a \\
\hline Espécie & TP & AR & VERM & TP + AR \\
\hline M. paraensis & $5,90 \pm 1,89$ a & $3,45 \pm 0,86$ a & $7,28 \pm 0,49 \mathbf{b}$ & $5,10 \pm 0,18$ a \\
I. pourgonii & $6,13 \pm 0,78$ a & $3,60 \pm 0,49$ a & $7,12 \pm 0,49 \mathbf{b}$ & $5,12 \pm 0,39$ a \\
\hline
\end{tabular}

Médias seguidas de letras iguais na mesma linha e na mesma coluna, não diferem entre si ao nível de $5 \%$ de probabilidade pelo Teste de Tukey.

\section{DISCUSSÃO}

O substrato mais eficiente na emergência das espécies foi a vermiculita, ao qual promoveu a maior quantidade de plantas emergidas. Alvino e Raiol, (2007) ao avaliarem 0 efeito de substratos verificaram que a vermiculita também apresentou efeito positivo na germinação de sementes de Ochroma pyramidale (Cav. ex Lam.) Urb. Resultados similares também foram encontrados por Alves et al. (2008) e Mondo et al. (2008) quando observaram a maior emergência e o vigor de sementes de Erythrina velutina Willd. e maior germinabilidade em sementes de Parapiptadenia rigida Benth respectivamente.
A influência da vermiculita no processo de emergência está relacionada com suas características, pois este material retém alto teor de umidade, apresenta boa estrutura para o desenvolvimento radicular, alta capacidade de retenção de água, baixa densidade e bom arejamento entre as partículas, favorecendo a emergência da planta (Martins et al., 2011; Andrade et al., 2000). Todavia, Ferreira et al. (2013) relataram que a vermiculita reduziu a percentagem de germinação e sobrevivência de Myrcia cuprea O. Berg Kiaersk, nesse sentido, Bezerra et al. (2002) também verificaram resultado negativo deste material na velocidade de germinação e 
desenvolvimento de plantas de Momordia charantia L.

O conhecimento das características do substrato, associado à exigência das espécies, proporcionará certamente o sucesso no processo de emergência e desenvolvimento da planta. De acordo com Resende et al. (2011) a composição do substrato, é um fator que desempenha papel importante para o sucesso da germinação, emergência e o crescimento da planta.

Por outro lado, a mistura de substrato com terra preta e areia também promoveu boas condições de emergência para $M$. paraenses e I. bourgonii, sendo muito próximos ao da vermiculita. Figueredo et al. (2012) relatam que a mistura de substratos visa determinar o melhor padrão vital de cultivo, favorecendo assim, a germinação e desenvolvimento da planta. Este efeito pode estar relacionado com a peculiaridade da espécie para com o substrato, indicando que as sementes de diferentes espécies podem exigir diferentes condições físicas e químicas para atingir satisfatórios índices de germinação.

Para Smiderle e Minami (2001), na produção de mudas de espécies nativas, o substrato deve proporcionar retenção de água suficiente para permitir a germinação e, quando saturado, manter quantidades adequadas de espaços porosos para facilitar o fornecimento de oxigênio, indispensável no processo de germinação e desenvolvimento da muda. Por este motivo, é necessário utilizar diferentes substratos para determinar a melhor forma de propagação, multiplicação, emergência e desenvolvimento de plantas de várias espécies (Cavalcanti; Resende, 2006).

Portanto, é importante considerar determinadas características da planta para a escolha do melhor substrato na produção de mudas, como por exemplo, a exigência das espécies quanto ao substrato, a emergência e ao desenvolvimento posterior das mudas que podem ser diferentes (Guimarães et al., 2011).

\section{CONCLUSÃO}

Os substratos não influenciaram no tempo de emergência, enquanto que a maior quantidade de sementes germinadas foi proporcionada pela vermiculita que atua como excelente retentora de água por apresentar baixa densidade e boa qualidade estrutural. 
Ao Conselho Nacional de Desenvolvimento Científico e Tecnológico pelo apoio/Processo: 561808/2010-4.

\section{REFERÊNCIAS BIBLIOGRÁFICAS}

ALVINO, F. O.; RAIOL, B. P. Efeito de diferentes substratos na germinação de Ochrama pyramidale (Cav. ex. Lam.) Urb. (Bombacaceae). Ciência Florestal. v.17, n.1, p.71-75, 2007.

ALVES, E. U.; ANDRADE, L. A.; BARROS, H. H. A.; GONÇALVES, E. P.; ALVES, A. U.; GONÇALVES, G. S.; OLIVEIRA, L. S. B.; CARDOSO, E. A. Substratos para testes de emergência de plântulas e vigor de sementes de Erythrina velutina Willd. (Fabaceae). Semina, v.29, n.1, p.69-82, 2008.

ANDRADE, A. C. S.; SOUZA, A. F.; RAMOS, F. N.; PEREIRA, T. S.; CRUZ, A. P. M. Germinação de sementes de jenipapo: temperatura, substrato e morfologia do desenvolvimento pós-seminal. Pesquisa Agropecuária Brasileira, v.35, n.2, p.609-615, 2000.

AYRES, M.; JÚNIOR, M. A.; AYRES, D. L.; SANTOS, A. S. Bioestat 5.0 - Aplicações Estatísticas nas Áreas das Ciências Biológicas e Médicas. Belém: Sociedade Civil Mamirauá/MCT/Imprensa Oficial do Estado do Pará, 364p. 2007.

BEZERRA, A. M. E.; MOMENTE, V. G.; ARAÚJO, E. C.; MEDEIROS FILHO, S. Germinação de sementes e desenvolvimento de plântulas de melão-de-são-caetano (Momordica charantia L.) em diferentes ambientes e substratos. Revista Ciência Agronômica, v.33, n.1, p.39-44, 2002.

BRAGA JUNIOR, J. M.; BRUNO, R. L. A.; ALVES, E. U. Emergência de plântulas de Zizyphus joazeiro Mart. (Rhamnaceae) em função de substratos. Revista Árvore, v.34, n.4, p.609-616, 2010. 
CARAUTA, J. P. P.; DIAZ, B. E. Figueiras no Brasil. Rio de Janeiro: Editora UFRJ, p.212, 2002.

CAVALCANTI, N. B.; RESENDE, G. M. Efeito de diferentes substratos no desenvolvimento do mandacaru sem espinhos (Cereus hildemannianus K. Schum). Revista Caatinga, v.19, n.3, p.255-260, 2006.

CORRÊIA, M. P. Dicionário de plantas úteis do Brasil e das exóticas cultivadas. Ministério da Agricultura, IBDF, p.484-485, 1984.

FERREIRA, N. M. M.; SANTOS, J. U. M.; FERREIRA, A. M.; GURGEL, E. S. C. Germinação de sementes e morfologia de plântulas de Myrcia cúprea (O. Berg.) Kiaersk. (Myrtaceae) espécie da restinga com potencial de uso no paisagismo. Revista Brasileira de Arborização Urbana, v.8, n.1, p.27-38, 2013.

FIGUEREDO, F. G.; LIMA, C. T. M. C.; SANTOS, M. R.; PADOVAN, M. P.; PIVA, M.; PEREIRA, Z. V. Efeitos de diferentes substratos na germinação de amora-brava (Maclura tinctoria (L.) D. Don ex Steud. - Moraceae). Revista Brasileira de Agroecologia, v.7, n.2, p.1- 5, 2012.

GUimarÃes, I. P.; COELHO, M. F. B.; BENEDITO, C. P.; MAIA, S. S. S.; NOGUEIRA, C. R. S.; BATISTA, P. F. Efeito de diferentes substratos na emergência e vigor de plântulas de mulugú. Bioscience Journal, v.27, n.6, p.932-938, 2011.

JABUR, M. A.; MARTINS, A. B. G. Influência de substratos na formação dos porta-enxertos: limoeiro-cravo (Citrus limonia Osbeck) e tangerina-cleópatra (Citrus reshni Hort. ex Tanaka) em ambiente protegido. Revista Brasileira de Fruticultura, v.2, n.2, p.01-10, 2002.

LOPES, J. C.; PEREIRA, M. D. Germinação de sementes de cubiu em diferentes substratos e temperaturas. Revista Brasileira de Sementes, v.27, n.2, p.146-150, 2005.

MARTINS, C. C.; MACHADO, C. G.; CALDAS, I. G. R.; VIEIRA, I. G. Vermiculita como substrato para teste de germinação de sementes de barbatimão. Ciência Florestal, v.21, n.3, p.421-427, 2011. 
MEROTTO, J, A.; SANGOI, L.; ENDER, M.; GUIDOLIN, A. F.; HAVEROTH, H. S. A desuniformidade de emergência reduz o rendimento de grãos de milho. Ciência Rural, v.29, n.4, p.595-601, 1999.

MONDO, V. H. V.; BRANCALION, P. H. S.; CICERO, S. M.; NOVEMBRE, A. D. L. C.; NETO, D. D. Teste de germinação de sementes de Parapiptadenia rigida (Benth.) Brenan (Fabaceae). Revista Brasileira de Sementes, v.30, n.2, p.177-183, 2008.

RESENDE, S. V.; CREPALDI, I. C.; PELACANI, C. R.; BRITO, A. L. Influência da luz e substrato na germinação e desenvolvimento inicial de duas espécies de Calliandra Benth. (Mimosoideae - Leguminosae) endêmicas da chapada diamantina, Bahia. Revista Árvore, v.35, n.1, p.107-117, 2011.

SMIDERLE, O. S.; MINAMI, K. Emergência e vigor de plântulas de goiabeira em diferentes substratos. Revista Científica Rural, v.6, n.1, p.38-45, 2001.

SOUSA, M. P.; BRAGA, L. F.; BRAGA, J. F.; DE SÁ, M. E.; MORAES, M. L. T. Influência da temperatura na germinação de sementes de sumaúma (Ceiba pentandra L.) Gaertn. Bombacaceae. Revista Brasileira de Sementes, v.22, n.1, p.110-119, 2000.

SOUZA, E. R. B.; CARNEIRO, I. F.; NAVES, R. V.; BORGES, J. D.; LEANDRO, W. M.; CHAVES, L. J. Emergência e crescimento de cagaita (Eugenia dysenterica DC.) em função do tipo e do volume de substratos. Pesquisa Agropecuária Tropical, v.31, n.2, p.89-95, 2001.

SOUSA, J. S.; BASTOS, M. N. do C.; GURGEL, E. S. C. O gênero Inga (LeguminosaeMimosoideae) na Província Petrolífera de Urucu, Coari, Amazonas, Brasil, Rodriguésia, v.62, n.2, p.283-297. 2011.

VARELA, V. P.; COSTA, S. S.; RAMOS, M. B. P. Influência da temperatura e do substrato na germinação de sementes de itaubarana (Acosmium nites (Vog.) Yakovlev) Leguminosae, Caesalpinoideae. Acta Amazonica, v.35, n.1, p.35-39, 2005. 